\title{
Atypical synovial chondromatosis of the right knee: A case report
}

\author{
YU-PING YANG ${ }^{1 *}$, JIANG-JING WANG ${ }^{2 *}$ and HONG-YUAN LI ${ }^{3}$ \\ ${ }^{1}$ Institute of Sports Medicine, Peking University Third Hospital, Beijing 100191; ${ }^{2}$ Department of Orthopaedic Surgery, \\ Cangzhou Combinational Hospital of Chinese and Western Medicine, Cangzhou, Hebei 061001; \\ ${ }^{3}$ Department of Orthopaedic Surgery, Harrison International Peace Hospital, Hengshui, Hebei 053500, P.R. China
}

Received June 6, 2017; Accepted October 13, 2017

DOI: $10.3892 /$ etm.2018.5955

\begin{abstract}
Synovial chondromatosis, also known as synovial osteochondromatosis, is a rare, benign condition characterized by the formation of multiple cartilaginous nodules in the synovium of facet spaces. Synovial chondromatosis affects many joints, the knee being the most common. The present report examined a 47-year-old male with symptoms of swelling and pain in the right knee, who was admitted to hospital in September 2015. Following admittance, arthroscopic explorations were conducted. Viscous fluid and multiple cartilage-like clumps were identified in the patient's joints during surgery. There was evidence of synovial hyperemia and edema in the inner and outer lateral recesses of the patellar bursa, accompanied by villous projections. Synovium debridement and removal of cartilage-like free masses were performed. Following 6-month follow-up, the motion of the right knee ranged from $0-150^{\circ}$ and no further swelling or pain was experienced by the patient. Following arthroscopy, the international knee documentation committee function score improved from 70.6 to 89.4 points. The results of the present report indicate that arthroscopic exploration is an effective treatment for patients with synovial chondromatosis.
\end{abstract}

\section{Introduction}

Synovial chondromatosis, also known to as primary synovial chondromatosis, is a rare and benign disorder characterized by the presence of multiple cartilaginous nodules or loose bodies within the synovium of joints $(1,2)$. Synovial chondromatosis commonly presents in single large joints, including the knee, hip, elbow, shoulder and ankle (3). However, it may also affect smaller joints, including the distal radioulnar, tibio-fibular,

Correspondence to: Dr Yu-Ping Yang, Institute of Sports Medicine, Peking University Third Hospital, 49 North Garend Road, Haidian, Beijing 100191, P.R. China

E-mail: yupingyang956@163.com

*Contributed equally

Key words: synovial chondromatosis, knee joint, arthroscopic exploration, loose bodies metacarpophalangeal and metatarsophalangeal joints (4-7). Patients with synovial chondromatosis may either be asymptomatic or present with pain, swelling and restriction of movement (8). Clinical management of the condition commonly involves either surgical or arthroscopic procedures (9) to prevent further articular and peri-articular destruction and to eliminate symptoms (10). Synovial chondromatosis may be diagnosed using radiographs, computed topomography (CT) scans or magnetic resonance imaging (MRI), with definitive characteristics being determined by histological examination (11). However, radiography is the most commonly used diagnostic method, as it can identify mineralized nodules (12). The present report examined a case of knee synovial chondromatosis in a patient with atypical symptoms.

\section{Case report}

A 47-year-old male with no history of antecedent trauma presented with progressive pain in the right knee over a period of 4 months. Following 2 months worsening pain, the patient was admitted to Cangzhou Central Hospital (Cangzhou, China) in October 2014 for further examination and treatment. The patient's symptoms were insidious at onset and had progressed in severity over the preceding 4 months. Symptoms were exacerbated when the patient climbed stairs. In the 2 months prior to hospital admission, the patient exhibited aggravated swelling and pain of the right knee and experienced difficulties squatting and walking. The patient provided informed consent for the publication of the present case study. The current study was approved by the Ethics Committee of the Third Hospital of Peking University.

The patient received an X-ray, which identified diffuse swelling over the suprapatellar pouch and signs of effusion without evident loose bodies in the right knee. Additionally, a slightly high suprapatellar pouch density was identified, compared with the appearance of soft tissue shadows. However, bone-joint structures were normal (Fig. 1). On physical examination, there was marked swelling accompanied by local tenderness at the front and back of the right knee. The activity of the knee was limited to $10-130^{\circ}$ and the results of a floating patella test were positive. MRI scans identified a cartilage defect in the patella femoral condyle and multiple cystic necroses in the articular surface. In addition, low density loose radiodense bodies were observed in the suprapatellar bursa. Low density mass opacities were also identified in the infrapatellar fat pad 
of the knee and the posterior aspect of the femoral condyle. Effusive and synovial hypertrophy was detected behind the femoral condyle (Figs. 2 and 3). Following the diagnosis of synovitis $(13,14)$, the Chinese traditional medicine synovitis granule (GMP: Z13020929, Hebei Shenwei Pharmaceutical Co., Ltd., Zhangjiakou, Hebei, China) was administrated to the patient via oral administration three times $(24 \mathrm{~g})$ a day for 14 days. However, the patient's condition did not improve.

To further investigate the characteristics of identified masses and to aid the removal of lesions, arthroscopy surgical management was conducted using arthroscopy $\left(4 \mathrm{~mm} ; 30^{\circ}\right)$ and electric planer systems (Stryker, Kalamazoo, MI, USA). Arthroscopy was performed under combined spinal-epidural anesthesia and pneumatic tourniquets were used at limb roots. With liquid perfusion by pressure (15), the alternate arthroscopy, probe hooks and forceps were used to examine joint spaces and structures in the anteromedial and anterolateral direction. A yellow viscous joint effusion $\sim 10 \mathrm{ml}$ observed in the patient was extracted from the sheath of arthroscopy and a cartilage mass measuring $\sim 15 \times 20 \mathrm{~mm}$ was exposed in the bursae suprapatellaris (Fig. 4). The cartilage mass exhibited porcelain white surface, nodular shape and hard texture. Loose bodies were removed and residue was cleared. Marked hyperemia and edema were observed in the bursae suprapatellaris and in the medial and lateral recesses of the synovium, and were accompanied by villous projections. In addition, several white crystalline cartilages wrapped in synovial membranes were present, with an outer diameter of $\sim 1 \mathrm{~mm}$ (Fig. 5). Certain synovial membranes were selected for pathological analysis and all synovial membranes with hyperemia and edema were removed. Stage IV cartiliage abrasions measuring $\sim 10 \times 20 \mathrm{~mm}$ and $5 \times 20 \mathrm{~mm}$, were identified in the medial patella and medial trochlear, respectively (16) (Fig. 6). These were subsequently removed following identification. Irregular cartilage-like clumps $\sim 25 \times 20 \mathrm{~mm}$, with similar properties to bursae suprapatellaris loose bodies, were identified below the patellar fat pad and subsequently removed using forceps and a paner. A pedicle was attached to the fat pad of the knee for pathological examination. A third degree cartilage abrasion measuring $\sim 10 \times 20 \mathrm{~mm}$ was detected on the condylus medialis femoris. Medial and posterolateral incisions of the knee were performed, which identified cartilage-like masses measuring $25 \times 15 \mathrm{~mm}$ and $15 \times 15 \mathrm{~mm}$ in the internal and external joints, respectively (Figs. 7 and 8). The cartilage-like masses were removed and the joint was washed and the wound was subsequently closed. The patient was orally administrated anti-coagulant drug (Rivaroxaban, Schering AG, Berlin, Germany) $10 \mathrm{mg}$ per day for 12 days post-operatively and was instructed to complete strengthening exercises on the second day following treatment (17). Post surgery, ophthalmic forceps were used to separate the synovial layer and fiber layer of joint capsule and the synovial tissue was peeled off. The synovial tissue was then cut off using ophthalmic scissor and fixed in $10 \%$ formaldehyde for $24 \mathrm{~h}$ at room temperature. The fixed synovial tissue was embedded with paraffin for $30 \mathrm{~min}$ and sliced into sections 3-5 $\mu \mathrm{m}$ thick. Slices were de-waxed with xylene for 5-10 min and successively washed using alcohol (Sigma-Aldrich; Merck KGaA) in concentrations of 100, 95, 85 and 70\% (2-5 min for each step). Slices were then stained with hematoxylin for 5-10 min and eosin

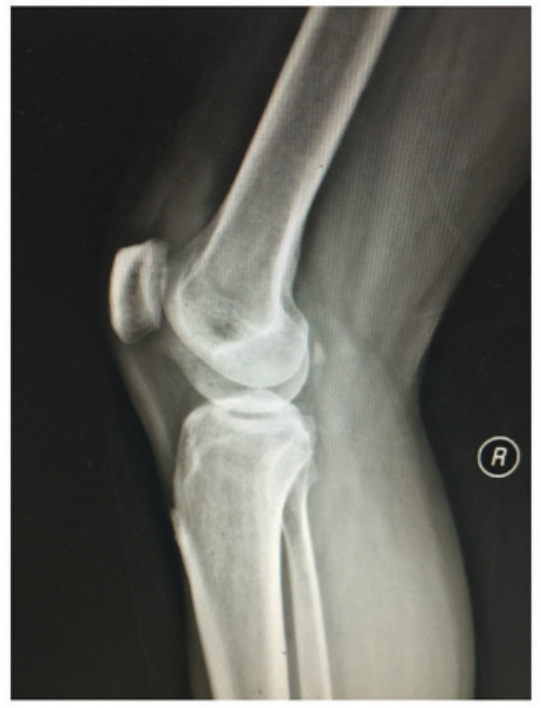

Figure 1. X-ray of the right knee pre-surgery.

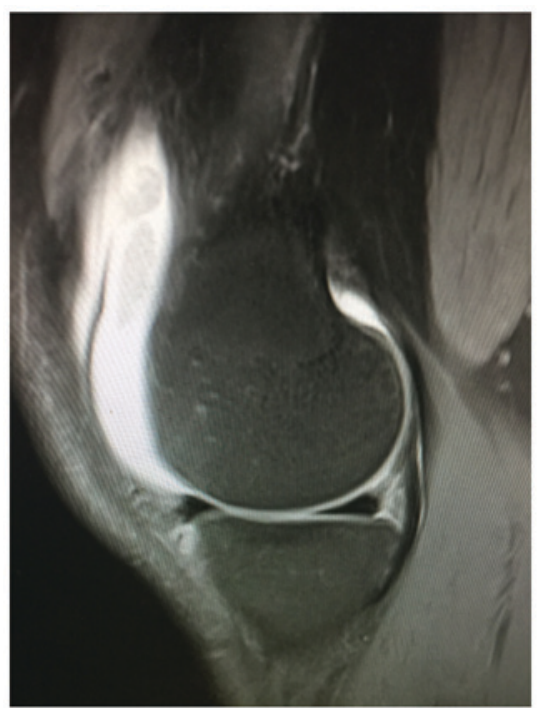

Figure 2. Sagittal T2-weighted magnetic resonance imaging.

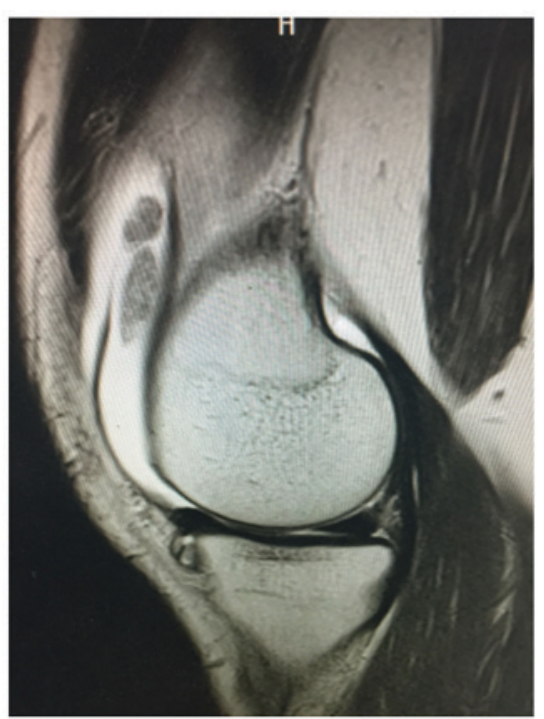

Figure 3. Sagittal T1-weighted magnetic resonance imaging. 


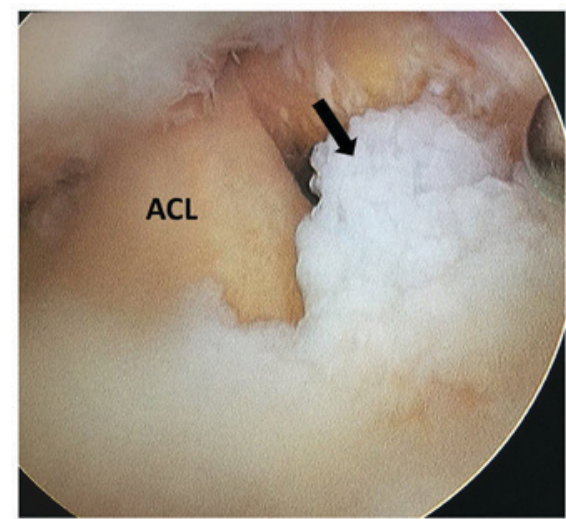

Figure 4. Knee synovial chondromatosis tumors. The black arrow indicates the synovial chondromatosis tumor located in front of the intercondylar fossa. ACL, anterior cruciate ligament.

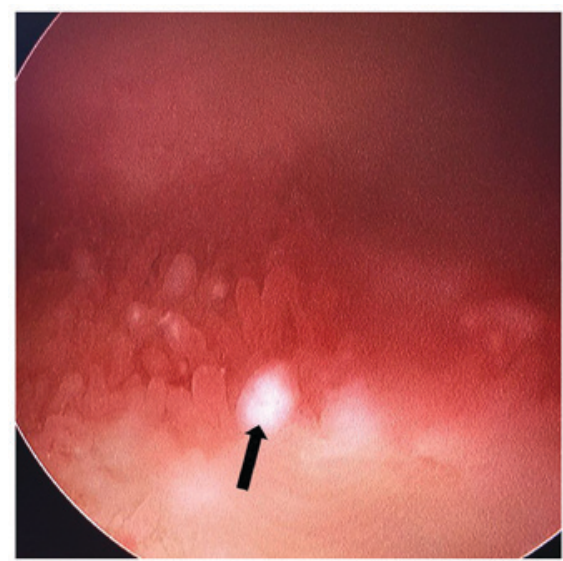

Figure 5. Evident hyperemia and edema were observed in the bursae suprapatellaris and there were several white crystalline cartilages wrapped in synovial membranes. The black arrow indicates the immature white tumor in the proliferated synovial membrane.

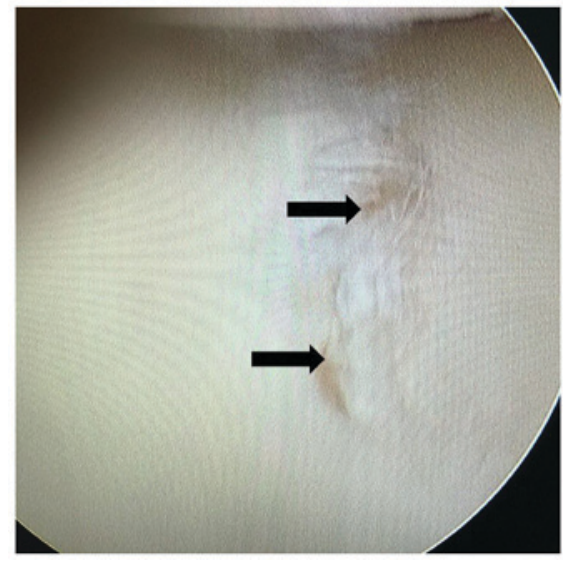

Figure 6. Trochlear cartilage injury in the lateral femur. The black arrows indicate the injury of the trochlear cartilage in IV degree, according to the Outerbridge classification (16) and the IV degree exhibited full thickness lesions.

for 1-3 min and then visualized using an optical microscope (magnification, x100; Olympus BX43, Olympus Corporation, Tokyo, Japan).

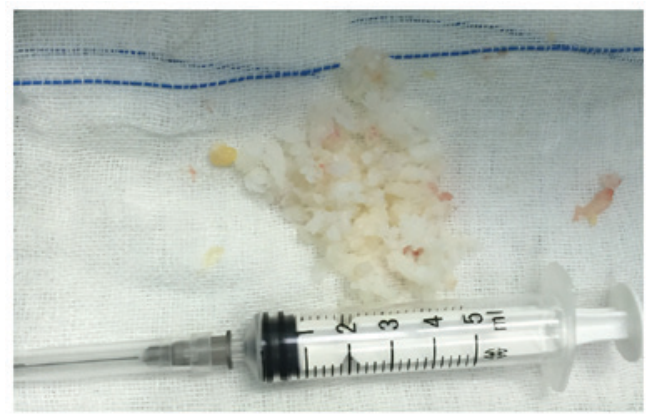

Figure 7. Macroscopic appearance of multiple loose bodies removed from the suprapatellar bursa.

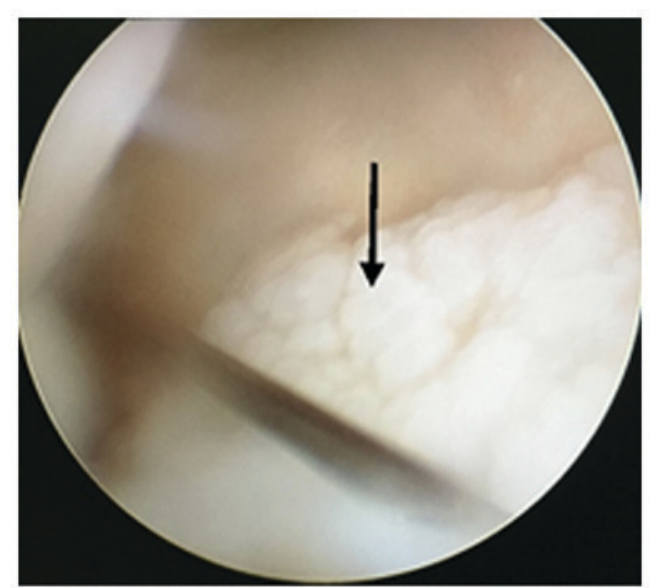

Figure 8. Arthroscopy identified multiple white loose bodies in the posterior-medial joint space. The black arrow indicates the synovial chondromatosis tumor in the posterior-medial joint space.

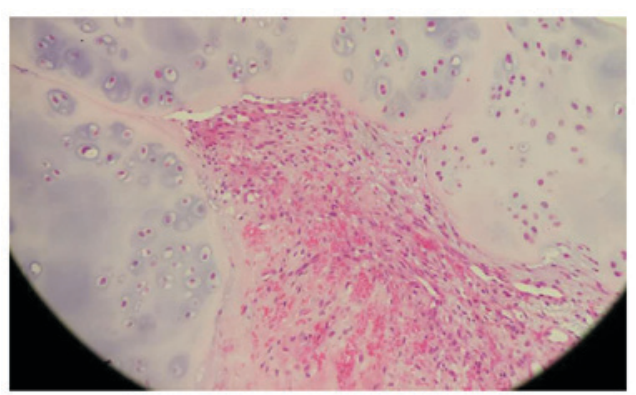

Figure 9. Synovial hyperplasia, synovial cartilage island formation and massive free bodies were observed in the synovium (Hemotoxylin and eosin staining; magnification, x100).

Post-operative pathological analysis revealed synovial hyperplasia cartilage island formation, and multiple white loose bodies were also observed in the joint space, which was consistent with synovial chondromatosis (Fig. 9). Following treatment, there was a 6-month follow up period in which the patient attended a follow-up session once a month for observations of the symptoms and the recovery state and movement range of the joint; no drugs were used during this time. And the patient's range of movement increased to $0-150^{\circ}$ flexion without pain during the 6-month follow up period. There was no recurrence of swelling or pain in the knee and 
the international knee documentation committee function score (18) was markedly increased post-surgery (from 70.6 to 89.4 points).

\section{Discussion}

Synovial chondromatosis is a disease with an unknown etiology that is potentially caused by the metaplasia of synovial cartilage (19). The formation of cartilaginous nodules or loose bodies in synovial tissues and joint cavities have impacts on subintimal fibroblasts in the tendons and bursae of synovial joints (20). These nodules may extrude from the synovium and either become loose bodies that float in the synovium or extend into the extra articular soft tissue of joints (21). According to Milgram's study, synovial osteochondromatosis may be classified in three stages based on gross and pathological findings. During stage I, active lesions of the synovium are present without loose bodies and synovial cartilage metaplasia may be observed using a light microscope. During stage II, transitional lesions with active intrasynovial proliferation and free loose bodies may be identified. Loose bodies also remain within the proliferated synovial membrane. In stage III, multiple loose bodies are released into the joint space and synovitis subsides (2). Partial or total synovectomy should be performed during stages I and II and free bodies should be removed at stage III without treatment for synovialis. The patient included in the present case report presented with stage II synovial osteochondromatosis. Cartilaginous nodules were removed and a partial synovectomy was performed in lesion areas.

Synovial chondromatosis commonly presents when patients are aged 30-50 years (22), however, it may also occur during childhood (23). The patient in the current case report presented with exhibited a relatively large and immature tumor present near the lower end of the anterior cruciate ligament, causing. Therefore, the patient exhibited a restriction of movement restriction. Furthermore, arthroscopy indicated that the diameter of the tumor was $>0.5 \mathrm{~cm}$ and the patient's loose bodies did not exhibit interlocking symptoms. Interlocking symptoms are not usually associated with free bodies and are instead associated with the size and location of free bodies, suggesting that the diagnosis of synovial chondromatosis should not be neglected for patients that who lack interlocking symptoms. Regular routine examinations, including frontal and lateral knee X-ray plates, CT and MRI scans may therefore be necessary to diagnose synovial chondromatosis.

Atypical symptoms of synovial chondromatosis are commonly confused with synovitis. Multiple circular loose bodies are usually detected in typical synovial chondromatosis using X-ray or CT scans (24). In the present report, no evident loose bodies were detected in the patient, apart from effusive radiodense areas in bursae suprapatellaris. Thus, the patient was initially diagnosed with early stage synovitis. MRI was performed following ineffective treatment with synovitis granule. It identified a large effusion and synovial hypertrophy behind the femoral condyle and mass shadows were observed in the anterior and posterior capsules of the intercondylar fossa. MRI scans are therefore a good method of diagnosing and characterizing synovial lesions, due to their high resolution of soft tissue $(25,26)$ and non-calcified cartilage nodules. The application of MRI in early phase synovial chondromatosis has definite auxiliary diagnostic value and enables the early detection and treatment of synovial chondromatosis.

Arthroscopy has gradually replaced traditional incision surgery, as it only requires a small incision and causes little pain; patients exhibit fast post-operative recovery and athroscopy is a more effective treatment than loose body removal alone $(27,28)$. Urbach et al $(29)$ reported that loose body removal, combined with localized synovectomy, was able to completely eliminate abnormal synovial tissue and prevent recurrence.

In conclusion, the present case report describes a rare case of synovial chondromatosis, successfully treated using knee arthroscopy. The present study indicated that the arthroscopic technique is a safe and effective method of treating synovial chondromatosis and may be developed as a routine treatment in the future.

\section{Acknowledgements}

This study was supported by The Fund Program for the Excellent Returned Overseas Chinese Scholars from Peking University Third Hospital (grant no. 76435-01).

\section{References}

1. Chiba S, Koge N, Oda M, Yamauchi R, Imai T, Matsumoto H and Yokogushi K: Synovial chondromatosis presenting with cervical radiculopathy: A case report. Spine (Phila Pa 1976) 28: E396-E400, 2003.

2. Milgram JW: Synovial osteochondromatosis: A histopathological study of thirty cases. J Bone Joint Surg Am 59: 792-801, 1977.

3. Roberts D, Miller TT and Erlanger SM: Sonographic appearance of primary synovial chondromatosis of the knee. J Ultrasound Med 23: 707-709, 2004.

4. von Schroeder HP and Axelrod TS: Synovial osteochondromatosis of the distal radio-ulnar joint. J Hand Surg Br 21: 30-32, 1996.

5. Batheja NO, Wang BY, Springfield D, Hermann G, Lee G, Burstein DE and Klein MJ: Fine-needle aspiration diagnosis of synovial chondromatosis of the tibiofibular joint. Ann Diagn Pathol 4: 77-80, 2000.

6. Warme BA, Tigrani DY and Ward CM: Metacarpophalangeal joint synovial osteochondromatosis: A case report. Iowa Orthop J 28: 91-93, 2008.

7. Taglialavoro G, Moro S, Stecco C and Pennelli N: Bilateral synovial chondromatosis of the first metatarsophalangeal joint: A report case. Reumatismo 55: 263-266, 2011 (In Italian).

8. Evans S, Boffano M, Chaudhry S, Jeys L and Grimer R: Synovial chondrosarcoma arising in synovial chondromatosis. Sarcoma 2014: 647939, 2014.

9. Yu GV, Zema RL and Johnson RW: Synovial osteochondromatosis. A case report and review of the literature. J Am Podiatr Med Assoc 92: 247-254, 2002.

10. NeumannJA, Garrigues GE, Brigman BEandEward WC: Synovial Chondromatosis. JBJS Rev 4: pii: 01874474-201605000-00005, 2016.

11. Varol A, Sencimen M, Gulses A, Altug HA, Dumlu A and Kurt B: Diagnostic importance of MRI and CT scans for synovial osteochondromatosis of the temporomandibular joint. Cranio 29: 313-317, 2011.

12. Xie S, Nevis J and Lezmi S: Pathology in practice. Chondro-osseous metaplasia consistent with synovial chondromatosisin a great horned owl. J Am Vet Med Assoc 245: 767-769, 2014.

13. Jiang W: Clinical observation of conbined treatment of traditional chinese medicine and western medicine in 35 cases with traumatic synovitis of knee joint. World Health Digest 433-433, 2012. 
14. McQueen FM: The MRI view of synovitis and tenosynovitis in inflammatory arthritis: Implications for diagnosis and management. Ann N Y Acad Sci 1154: 21-34, 2009.

15. Mannami K: Influence of intraarticular continuous perfusion of physiological saline on chondrocytes. Nihon Seikeigeka Gakkai Zasshi 59: 573-580, 1985 (In Japanese).

16. Outerbridge RE: The etiology of chondromalacia patellae. J Bone Joint Surg Br 43-B: 752-757, 1961.

17. Parvizi J, Azzam K and Rothman RH: Deep venous thrombosis prophylaxis for total joint arthroplasty: American Academy of Orthopaedic Surgeons guidelines. J Arthroplasty 23 (7 Suppl): S2-S5, 2008.

18. Collins NJ, Misra D, Felson DT, Crossley KM and Roos EM: Measures of knee function: International knee documentation committee (IKDC) subjective knee evaluation form, knee injury and osteoarthritis outcome score (KOOS), knee injury and osteoarthritis outcome score physical function short form (KOOS-PS), knee outcome survey activities of daily living scale (KOS-ADL), lysholm knee scoring scale, oxford knee score (OKS), western ontario and McMaster universities osteoarthritis index (WOMAC), activity rating scale (ARS), and tegner activity score (TAS). Arthritis Care Res (Hoboken) 63 (Suppl 11): S208-S228, 2011.

19. Narasimhan R, Kennedy S, Tewari S, Dhingra D and Zardawi I: Synovial chondromatosis of the elbow in a child. Indian $\mathrm{J}$ Orthop 45: 181-184, 2011.

20. Surwade A, Chaudhary A and Mahale Y: Synovial chondromatosis of the knee: Management with arthroscope-assisted synovectomy and removal of loose bodies: A case report IJHSR: 6: 388-391, 2016

21. Birchall D, Khangure MS and Spagnolo DV: Vertebral synovial osteochondromatosis with compressive myelopathy. Spine (Phila Pa 1976) 24: 921-923, 1999.
22. Jesalpura JP, Chung HW, Patnaik S, Choi HW, Kim JI and Nha KW: Arthroscopic treatment of localized synovial chondromatosis of the posterior knee joint. Orthopedics 33: 49, 2010.

23. Kistler W: Synovial chondromatosis of the knee joint: A rarity during childhood. Eur J Pediatr Surg 1: 237-239, 1991.

24. Sato J, Notani KI, Goto J, Shindoh M and Kitagawa Y: Synovial chondromatosis of the temporomandibular joint accompanied by loose bodies in both the superior and inferior joint compartments: Case report. Int J Oral Maxillofac Surg 39: 86-88, 2010.

25. Frick MA, Wenger DE and Adkins M: MR imaging of synovial disorders of the knee: An update. Radiol Clin North Am 45: 1017-1031, 2007.

26. Rehm J, Zeifang $\mathrm{F}$ and Weber MA: Imaging of the elbow joint with focus MRI. Part 2: Muscles, nerves and synovial membranes. Radiologe 54: 279-294, 2014.

27. Samson L, Mazurkiewicz S, Treder M and Wiśniewski P: Outcome in the arthroscopic treatment of synovial chondromatosis of the knee. Ortop Traumatol Rehabil 7: 391-396, 2005.

28. Ogilvie-Harris DJ and Saleh K: Generalized synovial chondromatosis of the knee: A comparison of removal of the loose bodies alone with arthroscopic synovectomy. Arthroscopy 10: 166-170, 1994.

29. Urbach D, McGuigan FX, John M, Neumann W and Ender SA: Long-term results after arthroscopic treatment of synovial chondromatosis of the shoulder. Arthroscopy 24: 318-323, 2008. 